\title{
Plausible Intermediate Mass Black Hole from M82X-2 X-Ray Pulsations
}

\author{
Gagik Ter-Kazarian* and Saleh Al Shidhani \\ Phys. Dept.College of Science, SQU, POBox 36, Postal Code 123, Al-Khoud, OMAN \\ *Permanent address: Ambartsumian Byurakan Astrophysical Observatory \\ Byurakan 378433, Aragatsotn District, Armenia \\ Email: gago_50@yahoo.com
}

\begin{abstract}
Recently observed rare mighty X-ray coherent pulsations coming from M82X-2 impose a significant challenge in breaking the Eddington limit. Alternative approach to circumvent the obstacles was studied in $[1,2]$ by addressing the M82X-2 as a spinning intermediate mass black hole, resided in final stage of growth. This approach employs the microscopic theory of black hole $(\mathrm{MTBH})$, which explores a spontaneous breaking of gravitation gauge symmetry at huge energies. The preceding developments of this theory are proved to be quite fruitful for addressing the growth and merging phenomena of the black hole seeds. It explains as well the origin of ZeV-neutrinos, which are of vital interest for the source of ultra-high energy particles. In the present work, we further expose the assertions made in previous model via a computation of essential astrophysical corrections introduced by the rotation to the characteristic phase profile of M82X-2.
\end{abstract}

Keywords: Black hole physics; accretion: accretion discs; X-rays: binaries; X-rays: individual (NuSTAR J095551+6940.8).

\section{Introduction}

The accreting off-nuclear point sources in nearby galaxies, so-called the ultraluminous X-ray sources (ULXs), have attracted a great deal of observational and theoretical attention, in part because their luminosities suggest that they may harbor intermediate mass black holes (IMBHs), with an ubiquitous feature of the mass fits of more than $10^{2}-10^{4} M_{\odot}[3,4]$. Their luminosity ranges from $10^{39} \mathrm{erg} \mathrm{s}^{-1} \leq$ $L(0.5-10 \mathrm{keV}) \leq 10^{41} \mathrm{erg} \mathrm{s}^{-1}$, which exceeds the theoretical maximum for spherical infall (the Eddington limit) onto stellar-mass black holes. In spite of significant efforts in more than three decades since the discovery of ULXs, the astronomers have not yet clarified their nature. Assuming the emission is isotropic, in general, the extreme luminosities of ULXs suggest either the presence of IMBHs, see e.g. [5], or stellar-mass black holes $\left(M \leq 10 M_{\odot}\right)$ that are either breaking or circumventing their Eddington limit via somewhat peculiar geometric beaming of accretion flow [6], which remains still a relatively poorly understood regime.

The most bright in persistently X-rays source in the regions of nuclear and disk of the galaxy M82 (NGC 3034) is M82X-1 which can reach $L(0.3-10 \mathrm{keV}) \sim 10^{41} \mathrm{erg} \mathrm{s}^{-1}$. This source is the best known candidate for an IMBH, for which most evidence stacks up through a combination of its extreme luminosity, the $62.0 \pm 2.5$ day periodicity in the X-ray source, co-location within the young, dense stellar cluster MCG-11 with an age of 7-12 Myr [7]. The second brightest is being a transient, M82X-2, X-ray luminosity of which varies from below the detection level $2.5 \times 10^{38} \mathrm{erg} \mathrm{s}^{-1}$ to its active state $L(0.3-10 \mathrm{keV}) \simeq 1.8 \times 10^{40} \mathrm{erg} \mathrm{s}^{-1}$ and it was turned off twice in 1999 and 2000 indicating a factor of $>50$ variability. These two sources are separated by $5^{\prime \prime}$, and so can only be clearly resolved by the Chandra X-ray telescope. The X-ray spectra of some Chandra observations of M82X-2 are best fitted with an absorbed powerlaw model with photon index ranging from 1.3 to 1.7. These spectra are similar to those of Galactic black hole binary candidates seen in the low/hard state except that a very hard spectrum was seen in one of the observations.

Recently the NuSTAR group has detected the rare mighty X-ray coherent pulsations coming from the M82X-2 [8]. The source has the maximum luminosity $L(3-30 \mathrm{keV})=4.9 \times 10^{39} \mathrm{erg} \mathrm{s}^{-1}$, of average period $1.37 \mathrm{~s}$ and a 2.5 -day sinusoidal modulation. This, together with the spin-up behavior, indicative of 
an accretion torque unambiguously, allow to feature the M82X-2 as mass-exchange binary that contains a nondegenerate secondary donor star.

The proposals of more recent phenomenological studies (e.g. [9]-[14]) and references therein, which demonstrate either breaking or circumventing the Eddington limit via somewhat peculiar features of accretion flow onto a highly magnetised neutron stars, are widely quoted in literature and accepted as eminently reasonable. However, these approaches are strongly model dependent, and subject to many uncertainties. The physics is obscured by multiple arbitrary assumptions and proliferation of a priori free parameters involved, while a consistent complete theory would not have so many free parameters. As these studies are only dependent of geometry of accretion flow, they are indeed wholly ruptured when the accretion is ultimately inhibited, which holds in case of violation of the Eddington limit. Indeed, the persistent X-ray radiation from M82X-2 ultimately precludes the possibility of typical pulsars, because such a collimation $(\sim 100)$, which is comparable to that obtained for black holes, would be needed to explain M82X-2 as beamed radiation from neutron star. Also, the centroid of the persistent X-ray emission is between M82X-2 and M82X-1 [8], and if M82X-1 is indeed harbors plausible IMBH, (e.g. [3,5]), we expect the similarity of the persistent X-ray properties of the M82X-1 and M82X-2 to imply that the non-pulsed emission from the latter originates in the accretion disc, as it must do in the black hole M82X-1. Therefore, to reconcile the unusual high luminosity of X-ray pulsations of M82X-2 with the most extreme violation of the Eddington limit, the only other way is offered by a black hole.

But the coherent periodicity of M82X-2 obviously rules out the Schwarzschild or Kerr black holes (sect.3), because: (i) Classically, black holes are perfect absorbers but do not emit anything; their physical temperature is absolute zero. (ii) The spinning black holes are axisymmetric and have no internal structure on which to attach a periodic emitter. Orbital motion, whether modulating some emission mechanism directly or exciting short-period pulsations, would decay very quickly due to gravitational radiation. Here and throughout we refer to these models as the phenomenological black hole models (PBHMs), because their only observable integral parameters of total mass and angular momentum still have to be put in the theory by hand.

In order to circumvent the obstacles without the need for significant breaking of Eddington limit, in $[1,2]$ the M82X-2 is addressed as a spinning IMBH (SIMBH) by exploring the growth behavior of the black hole seeds (for an observational evidence see sect.2), in the framework of MTBH. The MTBH is the extension of the PBHM and rather completes it by exploring the most important processes of rearrangement of vacuum state and a spontaneous breaking of gauge symmetry in gravity, and thereof for that of rearrangement of vacuum state, at huge energies (see e.g. $[15,16]$ and references therein). Replacing a central singularity by the infrastructures inside event horizon, subject to certain rules, this theory makes room for the growth and merging properties of accreting black hole seeds. Consequently, the MTBH explains the origin of ZeV-neutrinos which are of vital interest as the source of ultra-high energy (UHE) particles.

In this framework, M82X-2 is assumed to be SIMBH resided in final stage of growth. The thermal blackbody X-ray emission, arisen due to the rotational kinetic energy of black hole, escapes from event horizon through the vista to outside world that detected as ultraluminous X-ray pulsations. The M82X-2 indeed releases $99.59 \%$ of its pulsed radiative energy predominantly in the X-ray bandpass $0.3-30 \mathrm{keV}$. The observed ultraluminous X-ray pulsations are further determined by the complicated transfer of X-ray photons from the surface of M82X-2 through regions of external accreting plasma.

The position angles being the parameters of a model function can be adjusted via nonlinear regression analysis to the approximate solution of overdetermined systems to best fit a data set from observed pulsed profile of M82X-2. This missing ingredient is a shortcoming of previous astrophysical model, which will be solved by iterative refinement. With this perspective in sight, in present article we further analyse and compute the essential astrophysical corrections, introduced by the rotation, to the characteristic phase profile of M82X-2. We hope that the microscopic model will allow us, further, to obtain the solutions to $\mathrm{X}$-ray pulsations from M82X-2. This would have a substantial impact on theoretical study of the coherent picture of the ULX Universe.

We will proceed according to the following structure. To start with, the next section deals with the observational aspects of important astrophysical phenomena of the growth and merging of black holes. In section 3, we give a brief review of the key objectives and assessment of growth and merging properties of black holes within phenomenological models. We provide a detailed analysis aimed at clarifying the 
current situation. We would like critically re-examine the past and present states, and attempt to chart the future of the subject. In section 4, the same issue is briefly accounted in the framework of MTBH, with a perspective of the preceding developments of the latter. Section 5 deals with the 4D geometry of stationary and axisymmetric space. A particular analysis of the rotating SPC is given in section 6 . The periodic source M82X-2 and the results of computation of the corrections, introduced by the rotation, to the characteristic phase profile of M82X-2 are presented in section 7 . The concluding remarks are given in section 8.

\section{Observational and Computational Aspects of Growth of Black Hole Seeds}

Significant progress has been made in the last few years on understanding how supermassive black holes form and grow. Given the current masses of $10^{6-9} M_{\odot}$, most black hole growth happens in the AGN phase. A significant fraction of the total black hole growth, 60\% [17], happens in the most luminous AGN, quasars. In an AGN phase, which lasts $\sim 10^{8}$ years, the central supermassive black hole can gain up to $\sim 10^{7-8} M_{\odot}$, so even the most massive galaxies will have only a few of these events over their lifetime. Aforesaid gather support especially from a breakthrough made in recent observational, theoretical and computational efforts on understanding of evolution of black holes and their host galaxies, particularly through self-regulated growth and feedback from accretion-powered outflows (see e.g. [18]-[21]). Whereas the multiwavelength methods are used to trace the growth of seed BHs, and the prospects for future observations are reviewed.

The observations provide strong support for the existence of a correlation between supermassive black holes and their hosts out to the highest redshifts. Particularly, the observations of the quasar luminosity function show that the most supermassive black holes get most of their mass at high redshift, while at low redshift only low mass black holes are still growing [22]. This is observed both in optical [23] and hard X-ray luminosity functions [22], which indicates that this result is independent of obscuration. Natarajan [20] has reported that the initial black hole seeds form at extremely high redshifts from the direct collapse of pre-galactic gas discs. Populating dark matter halos with seeds formed in this fashion and using a Monte-Carlo merger tree approach, he has predicted the black hole mass function at high redshifts and at the present time. The most aspects of the models that describe the growth and accretion history of supermassive black holes, and evolution of this scenario have been presented in detail by $[18,19]$. In these models, at early times the properties of the assembling black hole seeds are more tightly coupled to properties of the dark matter halo as their growth is driven by the merger history of halos. While a clear picture of the history of black hole growth is emerging, significant uncertainties still remain [21], and in spite of recent advances $[17,20]$, the origin of the seed black holes remains an unsolved problem at present.

\section{Assessment of Growth and Merging Properties of Black Holes within Phenomenological Models}

A general relativity (GR) has stood the test of time and can claim remarkable success, although there are serious problems of the energy-momentum conservation laws of gravitational interacting fields, the localization of energy of gravitation waves, the role of singularities of black holes, and also severe problems involved in quantum gravity. This state of affairs has not much changed up to present and proposed abundant models are not conductive to provide non-artificial and unique recipe for resolving these controversial problems. In the framework of GR, the PBHM implies the most general Kerr-Newman black hole model, with the only independent observable integral parameters of total mass $(M)$, angular momentum $(J)$ and charge $(Q)$. Note that, even in the vacuum, asymptotically flat, four dimensional case relatively little is known about stability of the solutions to Einstein's equations beyond the linear level. In particular, the Kerr solution has not been proved to be stable, although both linearized analytic calculations and numerical calculations indicate that it is (e.g. [24]).

A tacit assumption of theoretical interpretation of described in previous section astrophysical scenarios is a general belief reinforced by statements in textbooks, that a long-standing PBHM can describe the growth of accreting black hole seeds. But such beliefs are suspect and should be critically re-examined. 
For this reason, let us below collect and briefly review the necessary ideas behind the various specific constructions and suggestions on the conceptual problems of GR, the singularities and the thermodynamics of black holes in semiclassical and quantum physics. We would like to concentrate on the critical discussion of the past and present states, evaluating those strategies, approaches etc., that are explicitly and unambiguously given and applicable in any generic spacetime. This short review encompasses many discoveries which unlocked the mysteries or exposed some of the illusions of the considered field. Without it we cannot show how the matters stand, we almost bound of necessity to enter upon it, if we would write of them at all. It was far from being complete, and our claim here is not to discuss the problems considered in detail, but rather to give a collection of problems that are effectively or potentially related to interpretation of the growth and merging properties of black holes within the phenomenological model.

\subsection{Some Conceptual Problems Plagued GR}

Eventually, experimental gravitation is a major component of the field, characterized by continuing efforts to test the GR's predictions. GR certainly can claim remarkable success at the post-Newtonian level where the experiments have reached high precision, including the light deflection, the Shapiro time delay, the perihelion advance of Mercury, the Nordtvedt effect in lunar motion, and frame-dragging [25]. Thereby gravitational wave damping has been detected in an amount that agrees with general relativity to better than half a percent using the Hulse-Taylor binary pulsar system [26], also see subsequent observations of its energy loss [27]. A growing family of binary pulsar systems is currently yielding new tests focusing on strong gravity and gravitational waves. These experiments will search for new physics beyond GR at many different scales: the large distance scales of the astrophysical, galactic, and cosmological realms; scales of very short distances or high energy; and scales related to strong or dynamical gravity.

The geometrical interpretation of gravitation, having arisen from the dual character of the metrical tensor in its metrical and gravitational aspects, is a noteworthy result of GR. Although this has the advantage in solving the problems of cosmology, nevertheless such a distinction of the gravitational field among the fields yields the difficulties in the unified theories of all interactions of elementary particles, and in quantization of gravitation. Moreover, there are problems of energy-momentum conservation laws of gravitational interacting fields, the localization of energy of gravitation waves, the singularities or black holes, and also severe problems involved in quantum gravity. The well defined local energy-momentum density for the gravitational field may set the conceptual basis for the understanding of energy loss by gravitational radiation.

The difficulty for this is rooted in the weak principle of equivalence (WPE), i.e. the universality of free fall. The gravitational action only depends on the gravitational field, since any further background structure would be precluded by diffeomorphism invariance. Since the WPE can be used to get rid of the gravitational field on a given point of spacetime, a crucial conceptual and practical caveats are involved in the association of energy and angular momentum with the gravitational field. That is, Riemannian geometry in general does not admit a group of isometries, therefore, it is impossible to define energymomentum as Noether local currents related to exact symmetries. This has challenged validity of the concepts of energy and angular momentum, when one attempts to perform their straightforward extension to the gravitational field.

Such an approach rapidly meets important conceptual difficulties. Namely, the formulation of meaningful global or quasi-local mass and angular momentum notions in GR and in the particular context of black hole spacetimes always needs the introduction of some additional structure in the form of quasilocal quantities and quasi-symmetries that restricts the study to an appropriate subset of the solution space of GR. Although a remarkable surge of activity of investigations in this field has arisen recently, but the theory of quasi-local observables in general relativity is far from being complete. It is surprising that one has not only no ultimate, generally accepted expression for the energy-momentum and especially for the angular momentum, but there is no consensus in the relativity community even on general questions, for example, what should one mean by energy-momentum?

In the literature there are various, more or less ad hoc, lists of criteria of reasonableness of the quasilocal quantities (e.g. [28]). However, finding an appropriate quasi-local notion of energy-momentum has proven to be surprisingly difficult (for the comprehensive review see [29]). The situation is much less clear in the case of extended but finite spacetime domains, otherwise there are still controversies and 
open issues. For example, the Bartnik mass [30,31], which is a natural quasi-localization of the ADM mass, overestimates the physical quasi-local mass; or, the Hawking energy [32] and its slightly modified version, the Geroch energy [33], which are a well defined 2-surface observable, have not been linked to any systematic (Lagrangian or Hamiltonian) scenario. Similar situation holds for, e.g., the Penrose mass [34,35], Dougan-Mason energy-momenta [36], Brown-York-type expressions [37], etc, (for details see $[29])$.

The emphasis in modern gravitational research is on the fundamental questions at the intersection between particle physics and cosmology, including quantum gravity and the very early Universe. The GR as a geometrized theory of gravitation clashes from the very outset with basic principles of field theory. In accord to above said, this rather stems from the fact that Poincaré transformations no longer act as isometries, which posed severe problems in a Riemannian space interacting quantum field theory. The major unsolved problem is the non-uniqueness of the physical vacuum and the associated Fock space. A peculiar shortcoming is in the following two key questions to be addressed yet: (i) the absence of the definitive concept of space-like separated points, particularly, in the canonical approach, and the light-cone structure at each spacetime point; (ii) the separation of positive- and negative-frequencies for completeness of the Hilbert-space description. Due to it, a definition of positive frequency modes cannot, in general, be unambiguously fixed in the past and future, which leads to $\mid$ in $>\neq \mid$ out $>$, because the state $\mid$ in $>$ is unstable against decay into many particle $\mid$ out $>$ states due to interaction processes allowed by lack of Poincaré invariance. A non-trivial Bogolubov transformation between past and future positive frequency modes implies that particles are created from the vacuum and this is one of the reasons for $\mid$ in $>\neq \mid$ out $>$. This state of affairs has not much changed up to present and proposed abundant models are not conductive to provide non-artificial and unique recipe for resolving such controversies.

\subsection{Singularities}

Even though being among the most significant advances in astrophysics, it is rather surprising that PBHM is routinely used to explore the black hole growth and merging phenomena as though it cannot be accepted as convincing model for addressing this problem. Certainly, in this framework the very source of gravitational field of the black hole is a kind of meaningless curvature singularity at the central point of the stationary nonrotating $(J=0, Q=0)$ Schwarzschild black hole, or a ring singularity at the center of the rotating axisymmetric Kerr black hole, which are hidden behind the event horizon. The theory breaks down inside the event horizon which is causally disconnected from the exterior world. The Kruskal manifold is the maximal analytic extension of the Schwarzschild and Kerr solutions inside event horizon, so no more regions can be found by analytic continuation. But, the Kruskal continuation shows that the static observers fail to exist inside the horizon. This interior solution is not physically meaningful and essentially irrelevant.

Black holes then present a major challenge that they render time reversibility impossible. Objects thrown into a black hole can never be retrieved, because any timelike worldline must strike the central singularity which wholly absorbs the infalling matter. Any object that collapses to form a black hole will go on to infinite collapse to a singularity inside the black hole. This feature is interpreted either as black holes connect our world to other Universes via wormholes [38,39], or as an information thrown into a black hole can not be retrieved anymore. There is also an opposite view point that any object thrown into a black hole actually does leave some signals behind in own world $[40,41]$. Whatever it will be, in both cases the PBHM ultimately precludes any accumulation of matter inside event horizon and, thus, neither the growth of black holes nor the increase of their mass-energy density could occur at accretion of outside matter, or by means of merger processes.

Admitting an infinite collapse to the singularity inside the black hole as a physical law of Nature, it is impossible to answer, for example, what is further evolution of the coalescence and merger of binary black holes at grazing collision of members when, triggered by the emission of gravitational waves, their orbits will tighten by spiraling inwards? The nasty inherent appearance of black hole singularities, in fact, inhibits one to answer such purely academic questions. It is why excising the black hole interior, for example, is currently considered as an approximate solution to avoid singularities in dynamical simulations (e.g. [42]). 


\subsection{Black Hole Thermodynamics in Semiclassical Physics}

A current theoretical understanding of growth and merging behavior of black holes is based on the Hawking's theorem of surface area of a black hole [43,44]. Namely, in any interaction between matter or radiation with the black hole, the time dependent horizon area is never allowed to decrease with time. This is the meaning of the irreducible mass of the horizon, i.e. in a possible collision of several black holes, the surface area of the resulting merged black hole always exceeds the sum of the separate progenitor black holes. Say, if a black hole was being off the ordinary mass shell and carried no entropy, it would be possible to violate the law of energy conservation and $2^{\text {nd }}$ law of thermodynamics, because the energy and entropy in the exterior spacetime could be decreased by throwing matter into a black hole. In the framework of incomplete theory, therefore, the only way to maintain these laws there is nothing left but to admit stepwise, without any substantiation, that (i) the black hole resides on the ordinary mass shell $\left(E_{B H}=M_{B H} c^{2}\right)$ and (ii) it has entropy $\left(S_{B H}\right)$. Then the increase of these quantities may compensate the decrease of the energy and entropy carried by the mass that was swallowed. This is the meaning of the first and $2^{\text {nd }}$ laws of black hole dynamics [45]. The law of increase of area looks like the $2^{\text {nd }}$ law of thermodynamics for the increase of entropy, if one assigns an entropy to black hole that is proportional to its surface, and that the surface gravity stands for a temperature [46]. At first sight, this choice seems quite natural, but at closer inspection one finds that these intriguing ideas have encountered to severe objection: the entropy of a thermodynamic system is a measure of the large number of the real physical microstates that an observer would not be aware of when measuring macroscopic parameters, and so-called no hair theorems allow black hole, in best case, to have only a single microstate.

Classically, black holes are perfect absorbers but do not emit anything; their physical temperature is absolute zero. However, the spacetime associated to gravitational collapse to a black hole cannot be everywhere stationary. Therefore, in semiclassical geometric optics approximation, a particle creation determined by details of the collapse is allowed in non-stationary curved spacetime. This is a transient phenomenon because exterior spacetime is stationary at late times of existence of horizon independent of the details of the collapse. The infinite time dilation at the horizon of Schwarzschild black hole suggests a possible flux of such particles, which is the meaning of the Hawking radiation - the radiation seen by an observer in the space-time background of a Schwarzschild black hole when gravity will pull one of the members of pair into the black hole permanently, while the other assumed to be escaped from the black hole. Due to this radiation, a black hole that forms from gravitational collapse will eventually evaporate, after which the spacetime has no event horizon.

Continuation of the Schwarzschild metric to the Euclidean Schwarzschild metric implies that the non-singularity of the Euclidean metric is required for equilibrium. The quantum field theory (QFT) can be in equilibrium with a black hole only at the Hawking temperature, which is inversely proportional to the mass of black hole. Thereby the thermal equilibrium of a black hole with an infinite reservoir of radiation at Hawking temperature is unstable since if the black hole absorbs radiation its mass increases and its temperature decreases.

Similarly, the two features violate Hawking's area theorem: (i) in pair creation effectively a spacelike energy flux is involved - in contrast to the one of the essential postulates of the area theorem which requires that the energy-momentum tensor $T_{\mu \nu}$ should satisfy the dominant energy condition. This held if for all future-directed timelike vector fields $v$, the vector field $j(v) \equiv-v^{\mu} T_{\mu}{ }^{\nu} \partial_{\nu}$ is future-directed non-spacelike, or zero, i.e. no spacelike energy fluxes are allowed; (ii) the mass of black hole decreases during evaporation by energy conservation, as well as inevitably do the surface area and entropy.

Hawking radiation allows an interpretation of the laws of black hole mechanics as physically corresponding to the ordinary laws of thermodynamics. Having associated the entropy $S_{B H}:=\left[k c^{3} /(4 G \hbar)\right]$ $\times \operatorname{Area}(S)$ to the (spacelike cross section $\mathrm{S}$ of the) event horizon, the area theorem was replaced by a generalized $2^{\text {nd }}$ law (GSL) of thermodynamics, which includes the sum of the entropies of all black holes plus the entropy of matter in exterior spacetime [47]. The GSL provides means for the quantity $S_{B H}$ to be the physical entropy of a black hole. Notwithstanding it is possible to construct thought experiments (e.g. the so-called Geroch process) in which the GSL is violated, unless a universal upper bound $S_{m} / E \leq(2 \pi k / \hbar c) R$ for the entropy-to-energy ratio for bounded systems exists, where $E$ and $S_{m}$ are, respectively, the total energy and entropy of the system, and $R$ is the radius of the sphere that encloses the system $[48,49]$. 
A semi-classical method of modeling Hawking radiation as a tunneling of particles through a gravitational barrier has been developed in the framework of QFT on a curved gravitational background (e.g. [50,51] and references therein). Certain gravitational backgrounds gave rise to thermal radiation from the vacuum. This provides an alternate conceptual means for understanding the physics of cosmological pair production at a wide variety of cosmological event horizons in exotic spacetimes. However, all these processes for certain do not give physical insight regarding the nature of the microstates of a black hole and nor does it offer a substantiated reason for the black hole entropy $S_{B H}$. Moreover, in semi-classical analysis of the Hawking evaporation process, if the correlations between the inside and outside of the black hole are not restored during the evaporation process, then by the time that the black hole has evaporated completely, an initial pure state will have evolved to a mixed state, i.e., information will have been lost in the process of black hole formation and evaporation - the black hole information paradox (e.g. [25]). If information is lost into the black hole, which is ascribable to the propagation of the quantum correlations into the singularity within the black hole, this put QFT in curved spacetime in conflict with a basic principle of quantum mechanics [52], because of incompatibility with the unitary time evolution of a state vector in a Hilbert space. This violates the causality and energy-momentum conservation laws.

Some authors claim that the resolution requires an understanding of the Planck scale physics. Putting together the basic laws of physics, i.e. Heisenberg's uncertainty principle $\triangle p \triangle x \sim \hbar$, the existence of gravitating mass $E=m c^{2}$ and Schwarzschild radius $R_{g}=2 G m / c^{2}$ in Einstein's theory of gravity, these unambiguously assert the Planck's length $L_{P}:=\sqrt{\hbar G / c^{3}}=1.6 \cdot 10^{-33} \mathrm{~cm}$ to be a lower limit on the possible accuracy of position measurements (e.g. [53]). The Universe at the Planck scale is strong gravity where the Riemannian curvature of spacetime is comparable to the inverse square of a favorite Planck length scale. Another possible scale for strong gravity is the $\mathrm{TeV}$ scale associated with many models for unification of the forces, or models with extra spacetime dimensions.

\subsection{Black Hole Thermodynamics in Quantum Physics}

Stemming primarily from classical and semiclassical analyses, the discovery of the thermodynamic behavior of black holes has given rise to quantum physics occurring in strong gravitational fields. At the purely classical level, black holes within GR, of course, have nothing to do with the Planck scale quantum physics, because just outside the event horizon of an astrophysical black hole is weak gravity. Moreover, if pure states evolve to mixed states in a fully quantum treatment of the gravitational field, then at least the aspect of the classical singularity as a place where information can get lost must continue to remain present in quantum gravity. Nevertheless, the efforts to understand the mysterious statistical mechanical properties of black holes have led to many speculations about their quantum gravity origin. This in part is also due to the fact that the QFT in curved spacetime predicts an infinitely increase of a local temperature on the horizon of a black hole. This should not be believed when $k T$ reaches the Planck energy $(\sim \hbar c / G)^{1 / 2} c^{2}$ because quantum gravity effects cannot then be ignored and this temperature is then of the order maximum temperature in string theory. The latter appeals to GR as the low energy effective theory. Certainly, the quantum gravity is not needed to derive the black hole entropy, since it can be derived even from the general principles of a conformal field theory (CFT) on the horizon of the black holes (e.g. $[54,55])$.

However, black holes are localized objects, thus one must be able to describe their properties and dynamics even at the quasi-local level. The Schwarzschild black hole, fixing its temperature at infinity, has negative heat capacity. Similarly, in an asymptotically anti-de-Sitter spacetime fixing the black hole temperature via the normalization of the timelike Killing vector at infinity is not justified because there is no such physically distinguished Killing field [56]. These difficulties lead to the need of a quasi-local formulation of black hole thermodynamics. While the laws of black hole thermodynamics refer to the event horizon, which is a global concept in the spacetime, the subject of the recent quasi-local formulations is to describe the properties and the evolution of the so-called trapping horizon, which is a quasi-locally defined notion (e.g. [57]).

The area scaling character of the entropy perhaps implies a holographic principle $[58,59]$, formulated in the (spacelike) holographic entropy bound. This suggests that, at the fundamental (quantum) level, one should be able to characterize the state of any physical system located in a compact spatial domain by 
degrees of freedom on the surface of the domain too. This relation holds whenever holography dual of the QFT exists. In accord, the number of physical degrees of freedom in the domain is bounded from above by the area of the boundary of the domain instead of its volume, and the number of physical degrees of freedom on the 2D surface is not greater one-fourth of the area of the surface measured in Planck area units $L_{P}^{2}$. If $\Sigma$ be a compact spacelike hypersurface with boundary S, then the entropy $S(\Sigma)$ of the system in $\Sigma$ should satisfy $S(\Sigma) \leq k$ Area $(S) /\left(4 L_{P}^{2}\right)$. Formally, this bound can be obtained from the Bekenstein bound with the assumption that $2 E \leq R c^{4} / G$, i.e. that $R$ is not less than the Schwarzschild radius of $E$. Also, as with the Bekenstein bounds, this inequality can be violated in specific situations $[60,61]$. The origin of the holographic principle must lie in the number of fundamental degrees of freedom involved in a unified description of spacetime and matter $[62,61]$. This covariant entropy bound is much more quasilocal than the previous formulations, and is based on spacelike 2D surfaces and the null hypersurfaces determined by the $2 \mathrm{D}$ surfaces in the spacetime. Its classical version has been proved by [63].

Another quasi-local formulation of the holographic principle is suggested by [64]. Though not yet fully understood in general, the holographic principle is the key issue to the correspondence of anti-de Sitter spaces/conformal field theories (AdS/CFT) $[65,66]$. The AdS/CFT argues that the quantum gravity on $(\mathrm{d}+2)$-dimensional anti-de Sitter spacetime $\left(\mathrm{AdS}_{d+2}\right)$ is equivalent to a certain conformal field theory in $\mathrm{d}+1$ dimensions $\left(\mathrm{CFT}_{d+1}\right)$. By appealing to a duality between gravitational systems and conformal field theories, consequently the string theory seems to be able to count the described above microstates explicitly (e.g. [67]). In fact, the microstates are those due to entanglement of the vacuum of the black hole [68]. Indeed, one can always define the entanglement entropy in any quantum mechanical system. This is the entropy for an observer in the d-dimensional space-like submanifold A, in a given $(d+1)$ dimensional QFT, who is not accessible to B, which is a complement of $\mathrm{A}$, as the information is lost by the smearing out in region $\mathrm{B}$.

This origin of entropy looks analogous to the black hole entropy. That is, the microstates of the black hole are due to the entangled nature of the black hole vacuum, and are a result of an observer's inability to access the degrees of freedom that are hidden beyond the horizon. The subsystem B is analogous to the inside of a black hole horizon for an observer sitting in A, i.e., outside of the horizon. Indeed, this was the historical motivation of considering the entanglement entropy in QFT [66,69]. [70] argue that the entanglement mechanism is not specific to black holes but to any spacetime with a bifurcating Killing horizon.

For a comprehensive review of recent progresses on the holographic understandings of the entanglement entropy in the AdS/CFT correspondence, black hole entropy and covariant formulation of holography, see [71]. As notably pointed out by these authors, even after quite intense efforts in AdS/CFT for recent years, fundamental mechanism of the AdS/CFT correspondence still remains a mystery. In particular, one cannot answer which region of AdS is responsible to particular information in the dual CFT. There is also an essential discrepancy between the entanglement entropy and the black hole entropy, that the entanglement entropy is proportional to the number of matter fields, while the black hole entropy is not. The former includes ultraviolet divergences as opposed to the latter. So, due to existing discrepancies and absence of distinctive observationally tested predictions, there is no convincing reason to rely on the string theory in its present form.

\subsection{Cutting Short Where Our Analysis Is Leading to}

A principle feature that makes GR distinctively different from other field theories is the occurrence of singularities in spacetime. The singularities lead to regions of the Universe that cannot be observed. This causes an observer's inability to access the degrees of freedom that are hidden beyond the horizon which, in turn, leads to thermodynamical behavior of black holes. Notwithstanding, much remarkably efforts have been made in understanding of black hole physics, many important issues still remain unresolved. Primary among these are the black hole information paradox and issues related to the degrees of freedom responsible for the black hole thermodynamics.

Yet about 43 years after its conjecturing, solid physical information regarding the physical origin of black hole entropy is still lacking. Although no results on black hole thermodynamics have been subject to any experimental or observational tests, the attempts of theoretical interpretation of the black hole thermodynamics provide a basis for further research and speculation on the nature of its 
quantum gravitational origin. In the entanglement entropy and thermal atmosphere approaches, the relevant degrees of freedom are those associated with the ordinary degrees of freedom of quantum fields outside of the black hole.

The string theory implies weak coupling states, so it is not clear what the degrees of freedom of these weak coupling states would correspond to in a low energy limit where these states may admit a black hole interpretation. There is no indication in the calculations that these degrees of freedom responsible for black hole entropy should be viewed as being localized near the black hole horizon. As pointed out by [25], it is far from clear as to whether one should think of these degrees of freedom as residing outside of the black hole (e.g., in the thermal atmosphere), on the horizon (e.g., in Chern-Simons states), or inside the black hole (e.g., in degrees of freedom associated with what classically corresponds to the singularity).

At first sight described above choice for the definition of the laws of gravitation, and thereof for that of thermodynamics and entropy of black holes, seems quite natural, however, we do not share this view. It seems that the holographic principle, even at quantum level, indeed could not ultimately restore the complete information on the real physical state, but rather the elusive one, of any system located in a compact spatial domain by the degrees of freedom on the surface of the domain. Moreover, since there is no unique rigid notion of time translations in a classical general relativistic dynamics, the black hole entropy at least appears to be incompatible with any notion of ergodicity. This then ruptures the black hole entropy illusion which has insufficient dimensions. Only the complete internal solution was able to give a reliable information on the thermodynamic behavior and entropy of black hole, if and only if it is known deep within the black hole. Thus, it is premature to draw conclusions and only time will tell whether any of described above intriguing arguments is correct and actually realized in Nature.

Our misgiving about the views above also comes in part from a leading principle, that an appearance of singularities indicates only to the actual limits of validity of the theory, beyond which the laws of physics are violated. This we might expect to be reinforced by a robust intuition founded on past experience of simple physics. From this perspective, the aforementioned predictions on the black hole physics are then suspected to be only artifacts of incomplete theory. Consequently, a new conceptual framework will be required in order to have a proper understanding of the black hole physics.

Yet, it is still thought provoking how can one be sure that there should not be a physical law of Nature to prevent the gravitation theory to show such absurd tendencies of infinite collapse: say some hitherto unknown source of pressure may become important above extreme densities and energies for strong gravitation at very short distances when matter falls into central singularity as the collapse proceeds. Such a pressure may halt the infinite collapse. We believe that a complete, self-consistent gravitation theory will smear out singularities at huge energies via the most important spontaneous bricking of gravitation gauge symmetry. This will rather lead to phase transition of the matter to unknown yet state, with own energy scale, and physical entropy as a measure of the large number of the real thermodynamical microstates, which are compatible with the ergodicity. Whereas the central singularity, perhaps, will be replaced by the stable configuration composed of such matter, being hidden behind event horizon, therefore, can be the end product of the evolution of massive objects. This will ultimately circumvent a principle problem of an observer's inability to access the degrees of freedom that are hidden beyond the horizon, and a necessity to assign the elusive entropy to black hole.

Thus we conclude that PBHM, at least at its current state of development, is quite incapable of making predictions on growth and merging properties of the astrophysical black holes. One should therefore deliberately forebear from presumption of such behaviors, which seem nowhere near true if one applies the phenomenological model. That in this framework there is no provision for growth behavior of black holes, is because one assigns only an insufficient attributes to this. The PBHM is a rather restricted model and one needs to realise that if one can indeed gain insight into exploring a spontaneous breaking of gravitation gauge symmetry at huge energies, and thereof for that of rearrangement of vacuum state itself, one has then made room for growth and merging properties of black holes. This may shed further light upon the growth and merging phenomena of astrophysical black holes, that are in evidence throughout the Universe. To fill the void which the standard PBHM presents, one plausible idea to innovate the solution to alluded key problems would appear to be the MTBH. 


\section{MTBH: the Implications for IMBHs}

For the benefit of the reader, this section contains some of the necessary preliminaries on generic of the key ideas behind the MTBH, which one should know in order to understand the rest of the paper. The interested reader is invited to consult the original papers for further details (see e.g. [15,16] and references therein). For brevity reasons, we will not be concerned with the actual details of this comprehensive framework, but only use it as a backdrop to validate the theory with more observational tests.

Being an extension of PMBH, suitable for applications in UHE astrophysics, in this framework a substantial change of the properties of spacetime continuum, so-called inner distortion (ID), besides the curvature, arises at spontaneous breaking of gravitation gauge symmetry. The matter found in the ID-region of spacetime has undergone phase transition of the second order. In the resulting, so-called proto-matter, the pressure becomes dominant over gravitational force at very short distances when matter falls into central singularity as the collapse proceeds and, thus, it halts the infinite collapse. One of the most remarkable drawback of $\mathrm{MTBH}$ is that the central singularity cannot occur, which is replaced by finite though unbelievably extreme conditions held in the stable SPC where, nevertheless, static observers are existed. The SPC stood the tests of rigorous theoretical scrutinies of stability [72]. It was always found inside the event horizon, therefore it could be observed only in presence of accreting matter. The SPC, surrounded by the outside accretion disk, presents the microscopic model of AGN.

The stable SPC, in fact, is the end product of the evolution of massive objects. This makes room for growth and merging properties of black holes. This also ultimately circumvents the principle problem of an observer's inability to access the degrees of freedom that are hidden beyond the horizon, and a necessity to assign the misleading entropy to black hole. The physical entropy of SPC is a measure of the large number of the real thermodynamical microstates, which are compatible with the ergodicity. The SPC accommodates the highest energy scale up to hundreds $\mathrm{ZeV}$, which accounts for the spectral distribution of the resulting radiation of AGNs. An external physics of accretion onto the SPC in the first half of its lifetime is identical to the processes in Schwarzschild's model. A crucial difference comes in when one looks for the growth and merging behavior of black holes. To emphasize the distinction between phenomenological and microscopic black hole models, we present the schematic plots in Fig. 1, to guide the eye.
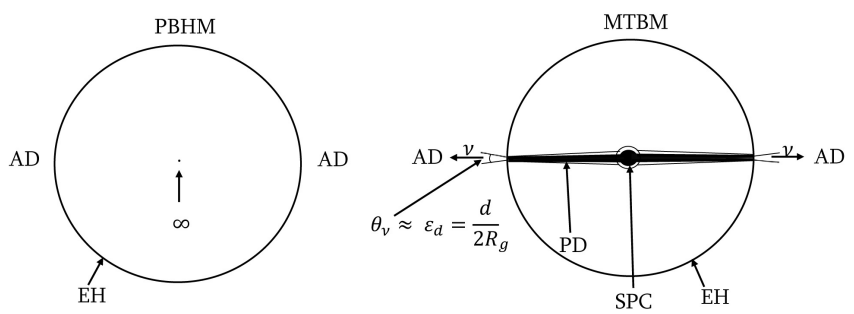

Figure 1. Left panel: Phenomenological model of non-spinning black hole. The meaningless singularity occurs at the center inside the black hole. Right panel: Microscopic model of non-spinning black hole, with the central stable SPC. An infalling matter with the time forms PD around the SPC. In final stage of growth, a PD has reached out the edge of the event horizon. Whereas a metric singularity inevitably disappears and UHE neutrinos may escape from event horizon to outside world through vista - a thin belt area $S=2 \pi R_{g} d$ - with opening angle $\theta_{\nu}$. Accepted notations: $\mathrm{EH}=$ Event Horizon, $\mathrm{AD}=$ Accretion Disk, $\mathrm{SPC}=$ Superdense Proto-Matter Core, $\mathrm{PD}=$ Proto-Matter Disk.

Understanding how seed black holes grow into IMBHs and SMBHs, has important implications for the duty-cycle of AGN, galaxy evolution, and gravitational wave astronomy. Therefore, a large series of numerical simulations are undertaken by $[15,16]$, with the goal to trace an evolution of the mass assembly history of 377 accreting SMBH seeds in AGNs to the present time and examine the observable signatures today.

The $\mathrm{ZeV}$-neutrino fluxes from plausible accreting SMBHs closely linking to the 377 AGNs are also calculated by $[15,16]$. Such neutrinos are produced in the medium of the SPC via simple (quark and 
pionic reactions) or modified $\mathrm{URCA}^{1}$ processes, even after the neutrino trapping. While hard to detect, the extragalactic $\mathrm{ZeV}$-neutrinos may reveal clues to the puzzle of the origin of ultra-high energy cosmic rays, as they have the advantage of representing unique fingerprints of hadron interactions and, therefore, can initiate via very complex chains of Z-burst interactions the cascades of UHE cosmic rays with energies exceeding $1.0 \times 10^{20} \mathrm{eV}$.

\section{$5 \quad$ Axisymmetric 4D Spacetime}

The non-spinning SPC is static and spherically symmetric. Therefore, we need further to be clear about more general geometry which can describe rotating axisymmetric SPC. The principle foundation of the spinning configurations comprises the following additional distinctive features with respect to nonspinning ones: (i) Rapid rotation causes the shape of the configuration to be flattened by centrifugal forces - flattened at poles and bulged at equator (oblate spheroid, which is second order effect in the rotation rate). (ii) A rotating massive configuration drags space and time around with it (non-Newtonian gravitational effect). The local inertial frames are dragged by the rotation of the gravitational field, i.e. a gyroscope orbiting near the configuration will be dragged along with the rapidly rotating configuration. This is probably the most remarkable feature that could serve as a link with the general description of spacetime. Beside the geodetic procession, a spin of the body produces in addition the Lense-Thirring procession.

The resulting bilinear form of norm $d \widetilde{s}^{2}$ of infinitesimal displacement on $V_{4}$ describes the metric of the stationary and axisymmetric space $V_{4}$ [2] in the most commonly used $3+1$ formalisms. In the $3+1$ formalism, as usual, $3+1$ foliations of spacetime $V_{4}$ by space-like 3-slices $\left\{\Sigma_{t}\right\}$ play an important role. The study of a dragging effect is assisted by incorporating with the soldering tools in order to relate local Lorentz symmetry to curved spacetime. These are the linear frames and forms in tangent fiber-bundles to the external general smooth differential manifold, whose components are so-called tetrad (vierbein) fields.

In the case of stationary and axisymmetric space $V_{4}$, the dimensionless potentials of the $1 \mathrm{D}$ gravitation filed $x_{0}$, the frame-dragging potential $x_{1}$, and the ID field $\bar{x}$, are generated by the stress-energy tensor of isolated spinning fluid $\widetilde{T} \rightarrow 0$ [2]. The existence of a global vacuum solution, $\chi\left(\equiv x_{0}, x_{1}\right)$, outside of the matter $(\bar{x}=0)$, amounts to solving the field equations in a nearly Newtonian weak source limit: $\widetilde{T} \rightarrow 0$. In Lorentz gauge, it is given by means of fundamental solution of Poisson's equation as a retarded integral of the form familiar from linearized field equation theory. The external metric $g^{\text {ext }}(\chi)$ is then written as

$$
\begin{aligned}
& g_{00}^{e x t}=\tau_{1}^{2}\left(1-\tan ^{2} \theta_{1}\right)+\tau_{2}^{2} \cos 2 \theta_{1}+\tau_{3}^{2}\left[\cos ^{2} \theta_{1}\left(1-x_{0}\right)^{2}-\sin ^{2} \theta_{1}\left(1+x_{0}\right)^{2}\right] \\
& g_{01}^{e x t}=-2 \tau_{1} x_{1}, \quad g_{11}^{e x t}=-\left(1+\tan ^{2} \theta_{1}\right), \quad g_{22}^{e x t}=-\cos 2 \theta_{1}, \\
& g_{33}^{\text {ext }}=-\cos ^{2} \theta_{1}\left(1+x_{0}\right)^{2}+\sin ^{2} \theta_{1}\left[\left(1-x_{0}\right)^{2}\right.
\end{aligned}
$$

where $\tan \theta:=-x_{1}$, and the parameters $\tau_{i}$ are directrix cosines $\left(\tau_{1}^{2}+\tau_{2}^{2}+\tau_{3}^{2}=1\right)$ of the "time" coordinate [2]. The two double principal null directions define "radially" ingoing and outgoing null congruences near the SPC which is the source of the field. The horizon is a $2 \mathrm{D}$ surface of spherical topology, where the redshift factor $(\alpha)$ vanishes

$$
g_{00}^{e x t}(r, \theta)=\alpha^{2}(r, \theta)=\tau_{1}^{2}\left(1-\tan ^{4} \theta_{1}\right)+\tau_{2}^{2}\left(1-\tan ^{2} \theta_{1}\right)+\tau_{3}^{2}\left[\left(1-x_{0}\right)^{2}-\tan ^{2} \theta_{1}\left(1+x_{0}\right)^{2}\right]=0 .
$$

The gravitational infinite redshift suppresses any emission at the horizon. The solution of (2), for given $x_{1}$, is

$$
x_{0}^{( \pm)}=\left(\tau_{3}\left(1-x_{1}^{2}\right)\right)^{-1}\left\{\tau_{3}\left(1+x_{1}^{2}\right) \pm \sqrt{\tau_{3}^{2}\left(1+x_{1}^{2}\right)^{2}-\left(1-x_{1}^{2}\right)\left(1-\tau_{2}^{2} x_{1}^{2}-\tau_{1}^{2} x_{1}^{4}\right)}\right\}
$$

where the discriminant has to be positive.

\footnotetext{
${ }^{1}$ G. Gamow was inspired to name the process URCA after the name of a casino in Rio de Janeiro, when M. Schenberg remarked to him that "the energy disappears in the nucleus of the supernova as quickly as the money disappeared at that roulette table".
} 
(i) At $x_{1}<1$, the gravitational field potentials $x_{0}^{( \pm)}$(3) yield two physical horizons. Their radii coincide with $r_{g}=R_{g} / 2\left(x_{0}^{( \pm)}=1\right)$ on the axis of rotation of SPC, located at $\theta=0$ (where $\left.x_{1}=0\right)$, where $R_{g}=2 G M / c^{2}=2.95 \times 10^{5} M / M_{\odot} \mathrm{cm}$ is the Schwarzschild radius (radius of a non-rotating black hole).

(ii) At $x_{1}\left(r_{0}\right)=1$, the outer oblate horizon can be formed only at infinity $r_{0} \rightarrow \infty$, where $x_{0}\left(r_{0} \rightarrow\right.$ $\infty) \rightarrow 0$.

(iii) For vanishing $x_{1} \rightarrow 0$, the solution (3), at $\tau_{3} \rightarrow 1\left(\tau_{2}=\tau_{1} \rightarrow 0\right)$, becomes static, spherically symmetric, yielding a single spherical event horizon $x_{0}^{( \pm)}(r) \rightarrow 1$ of previous model of non-rotating $\mathrm{SPC}([1])$.

The horizons are null surfaces because they are light-like. The hypersurfaces with $r_{ \pm}$are Killing horizons of the Killing vector field. A null hypersurface $\mathcal{N}$ is a Killing horizon of a Killing vector $\xi$ if, on $\mathcal{N}, \xi$ is normal to $\mathcal{N}[52]$. Suppose we adopt affine parametrization $l \cdot D l^{\mu}=0$. Since $\xi=f l$ on $\mathcal{N}$ for some function $f$, it follows that $\xi \cdot D \xi^{\mu}=k \xi^{\mu}$ on $\mathcal{N}$, where $k=\xi \cdot \partial \ln |f|$ is called the surface gravity. Since $\xi$ is normal to $\mathcal{N}$, Frobenius theorem implies that $\left.\xi_{[\mu} D_{\nu} \xi_{\rho]}\right|_{\mathcal{N}}=0$, where '[...]' indicates total anti-symmetry in the enclosed indices. For a Killing vector field $\xi$, it implies $D_{\mu} \xi_{\nu}=D_{[\mu} \xi_{\nu]}$. Except at points for which $\xi=0$, one then has $k^{2}=\left.(1 / 2)\left(D^{\mu} \xi^{\nu}\right)\left(D_{\mu} \xi_{\nu}\right)\right|_{\mathcal{N}}$. All points at which $\xi=0$ are limit points of orbits of $\xi$ for which $\xi \neq 0$, so continuity implies that this formula is valid even when $\xi=0$.

The external fields can be obtained straightforwardly in the weak source limit, where the spacetime is almost Minkowski $g \simeq \eta$. It is sufficient then to consider an isolated system only in the asymptotic linearized regime. Without loss of accuracy, one can therefore keep only leading linear order-terms of fields, ignoring a second and higher order effects. Linearized field equations guarantee conservation of 4-momentum and angular momentum of any body bounded by vacuum. Hence, in suitable asymptotically Minkowski coordinates, where the stationary Killing vector is given by $m=\partial_{t}$, the resulting redshift factor and the frame-dragging potential can be defined by means of the constants $M$ and $J$, respectively, as the total mass and intrinsic angular momentum of the source. These quantities are well substantiated by Komar integral [73]. That is, to every Killing vector field $\xi$ in the volume $V$ of spacetime on a spacelike hypersurface $\Sigma$, with boundary $\partial V$, one can associate the Komar integral.

The leading linear order-terms in asymptotic expansion of the redshift factor and the frame-dragging potential are given by

$$
\alpha\left(R_{g}, r\right) \simeq 1-\frac{R_{g}}{2 r}+O\left(\frac{1}{r^{3}}\right), \quad \omega(J, r)=\frac{2 G J}{c^{3} r^{3}}+O\left(\frac{1}{r^{4}}\right)
$$

Equating these values respectively to the functions $\left[g_{00}^{e x t}(M, r)\right]^{1 / 2}$ and $\left[-(1 / 2) g_{01}^{e x t}(J, r)\right]$, where the components of the external metric (1), which are accurate to linearized order, become $g_{00}^{\text {ext }} \simeq 1-2 \tau_{3}^{2} x_{0}$, and $g_{01}^{e x t}=-2 \tau_{1} x_{1}$, we finally obtain

$$
x_{0}(r)=\frac{R_{g}}{2 \tau_{3}^{2} r}, \quad x_{1}(r)=\frac{2 G J_{S P C}}{\tau_{1} c^{3} r^{3}} .
$$

The potential of dragging of inertial frames $\omega$ drops very rapidly with increasing radius (5). The maximal frame-dragging effects therefore can only be observed in the immediate vicinity of the event horizon:

$$
x_{1(H)}=\frac{\omega_{H}}{\tau_{1}} \simeq \frac{2 G J_{S P C}}{\tau_{1} c^{3} R_{g}^{3}}=\frac{J_{S P C} c^{3}}{4 \tau_{1} G^{2} M^{2}} .
$$

In physical units the angular momentum $J_{S P C}=a M_{S P C}$ becomes $J_{S P C}=a_{*} M_{S P C}\left(G M_{S P C} / c^{2}\right)$, such that $x_{1(H)}=\left(2 c a_{*} / \tau_{1} G M_{S P C}\right)$, where $a_{*}$ is the dimensionless angular momentum $\left|a_{*}\right| \leq 1$. The special case $a=M_{S P C}$ is the extreme solution. From this we get the maximal specific angular momentum $J_{S P C}^{\max }=\left(G M_{S P C} / c\right)$, and that $x_{1(H)}=2 a_{*} / \tau_{1} J_{S P C}^{\max }<1$

The Petrov type D vacuum solutions for stationary axisymmetric rotating SPC, therefore, satisfy the Robinson's theorem for Kerr solutions in vacuum [74]: the solutions, (i)-are asymptotically flat, (ii)contain a smooth convex horizon, (iii)- are nonsingular outside the horizon, and are uniquely specified by two parameters: the mass $M_{S P C}$ and angular momentum $J_{S P C}$. The angular velocity of a SPC is the sum of two terms: the classical one given by the intrinsic angular velocity $\Omega$ and the frame dragging $\omega$ from the rotation of absolute space. Near the horizon of SPC, for example, where the redshift tends to zero $(\alpha \rightarrow 0)$, the angular velocity of matter $\Omega$ is completely dominated by the frame-dragging effect. 
Whatever the intrinsic angular momentum of the incoming matter is, this matter is forced to rotate with the local angular velocity $\omega$, which is the maximal angular velocity at event horizon. When matter falls, say into a nonrotating black hole, it is forced to zero rotation near the horizon despite its angular momentum. The event horizon is a Killing horizon for the Killing field $\xi=k+\Omega_{H} m$, with $\xi^{2}=0$, where $\Omega_{H}$ denotes the angular velocity of the horizon as it is rigidly rotating.

Let $m=\partial_{t}$ be the axial Killing field, and $\widetilde{u}=\widetilde{u}^{t}\left(1, \Omega_{H}, 0,0\right)$ be the four-velocity of SPC. We may choose $\tau_{2}=0, \tau_{1}=\tau_{3}=1 / \sqrt{2}$. Since $x_{1(H)}<1$, analogous to the Kerr black hole, there are the outer horizon or event horizon by its radius $r_{+}$, and the inner horizon or Cauchy horizon by its radius $r_{-}$. The radii $r_{+}$and $r_{-}$coincide with $r_{g}\left(x_{0}^{( \pm)}=1\right)$ on the axis of rotation of SPC, located at $\theta=0\left(J_{S P C}=0\right)$. The oblate event horizon is the surface of the oblate spheroid with the semimajor $r_{+}$and semiminor $r_{g}$ axes, respectively. Dependent of the value of the potential $x_{1(H)}$, the Cauchy horizon either is the surface of the prolate spheroid with the semimajor $r_{g}$ and semiminor $r_{-}$axes, or oblate spheroid with the semimajor $r_{-}$and semiminor $r_{g}$ axes, respectively. The radii of the oblate event horizon and the Cauchy horizon read

$$
r_{\mp}:=r_{g} / \tau_{3}^{2} x_{0}^{( \pm)}
$$

where the potentials $x_{0}^{( \pm)}$are given by (3). These radii, as the function of $x_{1(H)}$, are plotted in Fig. 2 .

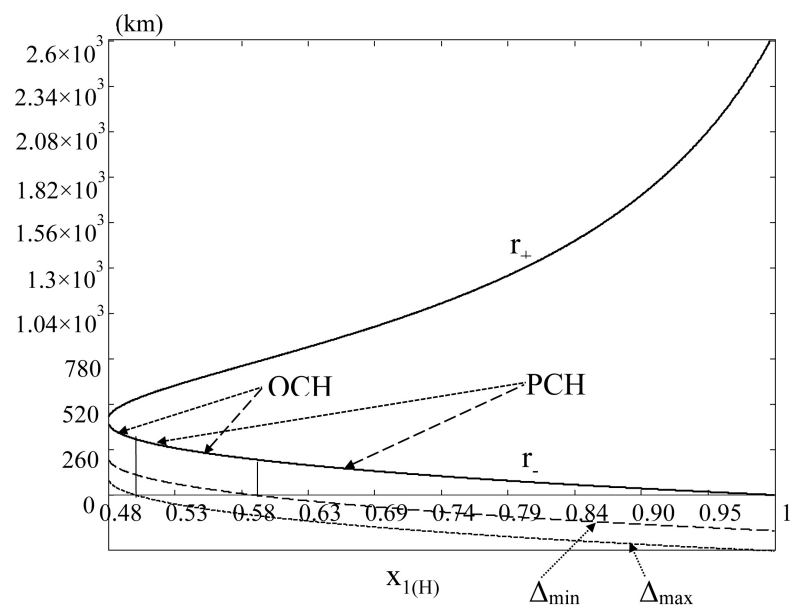

Figure 2. The radii $r_{ \pm}$of outer and inner horizons v.s. potential $x_{1(H)}$, for M82X-2, with the mass $M \simeq$ $138.5-226 M_{\odot}[1]$, i.e. $r_{g}=R_{g} / 2=r_{g}^{\max }-r_{g}^{\min } \simeq 204.3-333.4 \mathrm{~km}$.

Accepted notations: $\Delta_{\min }:=r_{-}-r_{g}^{\text {min }}, \Delta_{\max }:=r_{-}-r_{g}^{\max }$, OCH $:=$ Oblate Cauchy Horizon, PCH $:=$ Prolate Cauchy Horizon.

\section{Rotating SPC}

The entire set of the equations, describing the stationary and axisymmetric rotating SPC-configuration, includes the field equations, the hydrostatic equilibrium equation and the state equation of the sphericalsymmetric distribution of barionic matter in many-phase stratified states. A layering is a consequence of the onset of different regimes in equation of state. A rigorous numerical solution of these equations will be a separate topic of investigation elsewhere. But note that the key physical properties of SPC depend very little on the details of concrete SPC-model, as they are a direct consequence of the fundamental features of underlying gravitation theory. The latter explores a spontaneous breaking of gravitation gauge symmetry and a rearrangement of vacuum state. One therefore expects that the key properties of nonrotating SPC, outlined in section 3, even though without being carefully treated, retain for a rotating SPC too. 

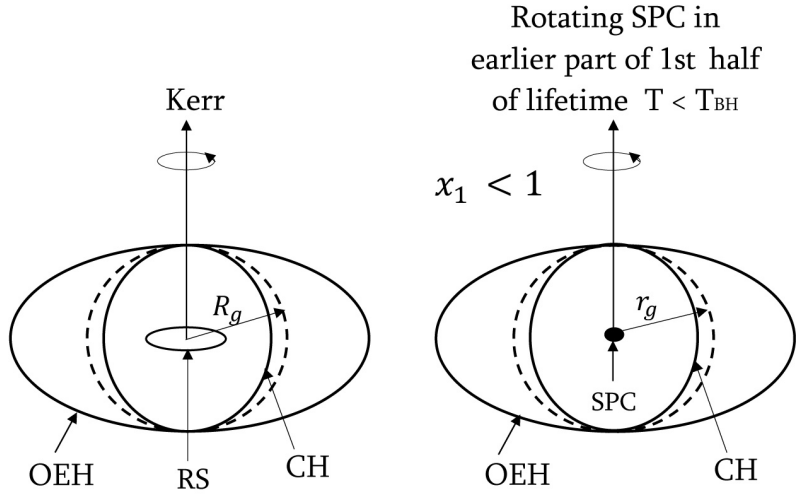

Figure 3. Left panel: Kerr model of spinning black hole. The meaningless ring singularity occurs at the center inside the black hole. Right panel: Microscopic model of rotating SPC in earlier part of first half of its lifetime $T<T_{B H}$. The picture is not to scale.

Abbreviated notations: OEH :=Oblate Event Horizon, SPC :=Superdense Proto-matter Core, RS :=Ring Singularity, $\mathrm{PCH}:=$ Prolate Cauchy Horizon.

So, in the first half of its lifetime, the external physics outside of outer oblate event horizon of accretion onto the SPC hole is very closely analogous to the processes in Kerr's model. But a crucial difference between Kerr and microscopic models is the interior solutions. However, the interior solution of MTBH is physically meaningful, because it has smeared out a central ring singularity of the Kerr black hole replacing it by the equilibrium SPC inside event horizon, where the static observers exist.

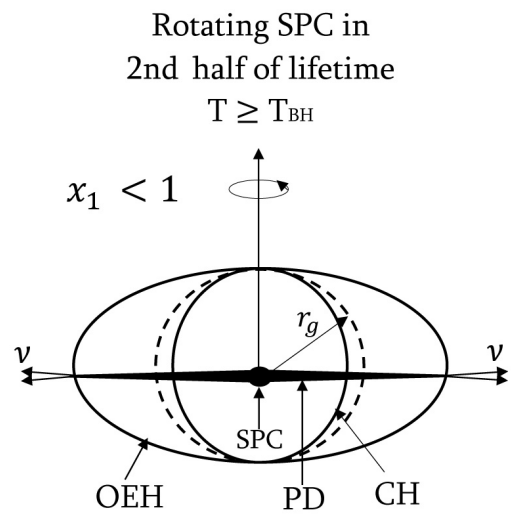

Figure 4. Microscopic model of rotating SPC in second half of its lifetime. An infalling matter already formed a thin co-spinning proto-matter disk which has reached out the edge of the outer oblate event horizon. A singularity inevitably disappears and the neutrinos escape to outside world through the vista.

Abbreviated notations: OEH $:=$ Oblate Event Horizon, SPC $:=$ Superdense Proto-matter Core, PCH $:=$ Prolate Cauchy Horizon, PD :=Proto-matter Disk.

Before attempting to build a physical model of the periodic ULX M82X-2, the other features of SIMBH configuration also need to be accounted. The event horizon is impenetrable barrier for crossing from inside the Kerr black hole. In the framework of microscopic model, this barrier disappears because a singularity at intersection of proto-matter disk with either Cauchy horizon or outer event horizon vanishes wherever the ID-field is not zero $(\bar{x} \neq 0)$, and hence the crossing of these horizons either from inside or outside of black hole at such conditions is allowed. Certainly, approaching to horizon the increase of particle concentration of infalling gas, $n$, is brought to halt when it asymptotically reaches to the 
threshold value, $n^{-1 / 3}=\lambda_{\bar{x}} \simeq 0.4 \mathrm{fm}$, and the ID-field $\bar{x}$ switches on and, thus, infalling gas becomes proto-matter. Then, a metric singularity vanishes as in the former case, and infalling gas passes freely through the horizon. The Fig. 4 emphasizes an apparent distinction between Kerr model and rotating $\mathrm{SPC}$ in second half of its lifetime.

\section{Periodic Source M82X-2}

With this guideline to follow, we may proceed to the real issue of the periodic ULX M82X-2. As pointed out by [1]: (i) the geodetic effect, as in the case of a gyroscope, leads an accretion stream to a tilting of its spin axis in the plain of the orbit. Hence a proto-matter disk will be tilted from the plane of accretion on a definite angle $\delta$ towards the equator.

Besides the UHE-neutrinos, produced in the deep interior layers of superdense proto-matter medium as in the previous non-spinning model, the additional thermal defuse blackbody radiation is released from the outer surface layers of ordinary matter of spinning SPC and co-spinning proto-matter thin disk. All of the rotational kinetic energy is dissipated as thermal blackbody radiation. This is due to the fact that these layers are optically thick and, eventually, in earlier half of the lifetime of spinning black hole, at times $<T_{B H}$, the strict thermodynamic equilibrium prevails for this radiation because there would be no net flux to outside of event horizon in any direction. That is, the emission from the isothermal, optically thick outer layers at surface is blackbody, which is the most efficient radiation mechanism. This radiation is free of trapping.

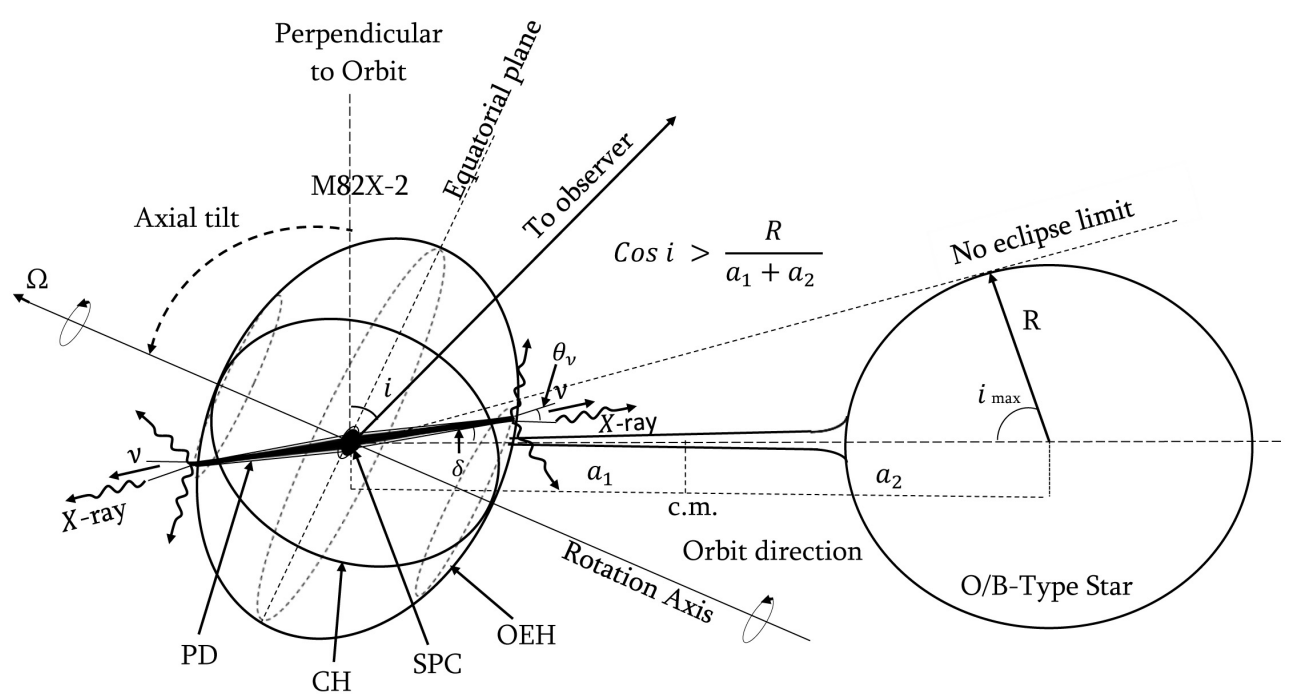

Figure 5. A schematic SIMBH model of M82X-2 constituting mass-exchange binary with the O/B-type donor star. The angle $i$ is the binary inclination with respect to the plane of the sky. No eclipse condition holds. In final stage of growth, PD has reached out the edge of the OEH. The thermal defuse blackbody X-rays beams may escape from SIMBH through a thin belt area $S=2 \pi R(\theta) d$ to outside world that sweep past Earth like lighthouse beacons. Parameters of a binary system is viewed in the orbital plane. The picture is not to scale.

Abbreviated notations: OEH $:=$ Oblate Event Horizon, SPC $:=$ Superdense Proto-matter Core, PCH $:=$ Prolate Cauchy Horizon, PD :=Proto-matter Disk.

The Fig. 5 schematically plots the SIMBH model of the periodic ULX M82X-2, constituting massexchange binary with the O/B-type donor star. The OEH-surface of the spheroid has the polar equation $R=R(\vartheta)$, where

$$
\frac{r_{g}^{2}}{R^{2}}=\cos ^{2} \vartheta+\left(1-e^{2}\right) \sin ^{2} \vartheta
$$


provided, $\vartheta$ is the reduced or parametric latitude $(-\pi / 2<\vartheta<\pi / 2)$, $e$ denotes the eccentricity $e:=$ $e\left(x_{1(H)}\right)=\sqrt{1-r_{g}^{2} / r_{+}^{2}\left(x_{1(H)}\right)}$, while $r_{+}\left(x_{1(H)}\right)$ and $r_{g}:=R(0)$ are the semimajor and semiminor axes, respectively, of the rotated ellipse of $\mathrm{OEH}$. The $\mathrm{OEH}$ introduces the corrections to the characteristic phase profile of previous model of X-ray radiation from M82X-2, derived in approximation of spherical EH [1]. Certainly, let $(\mathrm{z}, \mathrm{x}, \mathrm{y})$ be the space-fixed Cartesian coordinate system, with zx as a plane-of sight, and the axis $s$ of the M82X-2-fixed frame as the rotation axis. The angles $\theta$ and $\phi$ are spherical polar coordinates. The observed pulses are produced because of periodic variations with time of the projection on the plane-of sight, $d_{z x}(t)$, of the vector $\boldsymbol{d}(t)$ collinear to $\boldsymbol{n}(t)\left(\boldsymbol{d}(t)=d \frac{\boldsymbol{n}(t)}{|n(t)|}\right)$, where $\boldsymbol{n}(t)$ is the normal to the plane of the proto-matter disk at the moment $t$. The $\boldsymbol{n}(0)$ lies in the plane of zs. The pulsations will be observed if and only if the projection of the belt area $S_{z x}=C_{1}(e, \alpha) d_{z x}(d, \theta, \phi, \alpha, t)$ on the plane of sight zx is not zero, where $C_{1}(e, \alpha)$ is the circumference of the ellipse of intersection of the proto-matter disk plane with the $\mathrm{OEH}, \alpha$ is the angle between the equatorial plane and the proto-matter disk plane. The semi-major and semi-minor axes of this ellipse are $a_{1}=r_{+}:=R(\pi / 2), \quad b_{1}(\alpha):=R(\pi / 2+\alpha)$, respectively, such that

$$
e_{1}(\alpha)=\sqrt{1-b_{1}^{2}(\alpha) / r_{+}^{2}}=\frac{e}{\sqrt{1+\left(1-e^{2}\right) \cot ^{2} \alpha}} .
$$

Consequently, the phase profile can be recast into the form [2]:

$$
\begin{aligned}
& \Phi^{\prime}(e, \theta, \phi, \alpha, t)=\frac{2}{\pi \sqrt{1-e^{2}}} \int_{0}^{\pi / 2} \sqrt{1-e_{1}^{2}(\alpha) \sin ^{2} \gamma} d \gamma \Phi(\theta, \phi, \alpha, t)= \\
& \frac{1}{\sqrt{1-e^{2}}}\left[1-\Sigma_{n=1}^{\infty}\left(\frac{(2 n-1) ! !}{2^{n} n !}\right)^{2} \frac{e_{1}^{2 n}(\alpha)}{2 n-1}\right] \Phi(\theta, \phi, \alpha, t),
\end{aligned}
$$

where $n ! !$ is the double factorial $n ! !=\prod_{i=0}^{l}(n-2 i), l=[n / 2]-1$. Provided, the $\Phi(\theta, \phi, \alpha, t)$ is the phase profile in case of spherical EH

$$
\Phi(\theta, \phi, \alpha, t) \equiv \sqrt{1-\sin ^{2} \beta \sin ^{2}(\phi+A)} .
$$

Here the spherical triangle, with the lengths of three sides $\theta=(\widehat{z, s}), \alpha=(\widehat{s, n})$ and $\beta=(\widehat{z, n})$, is solved by the law of cosines

$$
\cos \beta(\theta, \alpha, t)=\cos \theta \cos \alpha+\sin \theta \sin \alpha \cos \Omega t, \quad \cos A(\theta, \alpha, t)=\frac{\cos \alpha-\cos \theta \cos \beta}{\sin \theta \sin \beta} .
$$

Using the relation (10), we may compute the corrections introduced by the rotation to the characteristic phase profile of M82X-2. The Fig. 6 - Fig. 8 are the examples, revealing the diversity of the behavior of corrected phase profiles versus the time, viewed at given position angles, for different values of eccentricity.

\section{Concluding Remarks}

In this work, we supplement previous investigations $[1,2]$ of observed unusual high luminosity of NuSTAR $\mathrm{X}$-ray pulsations from M82X-2, further, by numerical calculation of essential corrections introduced by the rotation to the characteristic phase profile of M82X-2. There are deep conceptual and technical problems involved, and these provide scope for the arguments discussed.

Exploring spontaneous breaking of gravitation gauge symmetry at huge energies, MTBH has smeared out a central singularity of black hole replacing it by the equilibrium SPC inside event horizon. This ultimately circumvents the principle problem, existing in $\mathrm{PMBH}$, of an observer's inability to access the degrees of freedom that are hidden beyond the horizon, and a necessity to assign the misleading entropy to black hole. The physical entropy of SPC is a measure of the large number of the real thermodynamical microstates, which are compatible with the ergodicity.

The oblate event horizon introduces the corrections to the characteristic phase profile of the previous model. Thereby the position angles can be adjusted via nonlinear regression analysis to the approximate solution of overdetermined systems to best fit a data set from observed pulsed profile of M82X-2. This missing ingredient is a shortcoming of previous astrophysical model, which will be solved by iterative refinement. For this aim, in present work we further analyse and extent a computation of the astrophysical corrections. We hope that the microscopic model will allow us, further, to obtain the solutions to X-ray pulsations from M82X-2. 

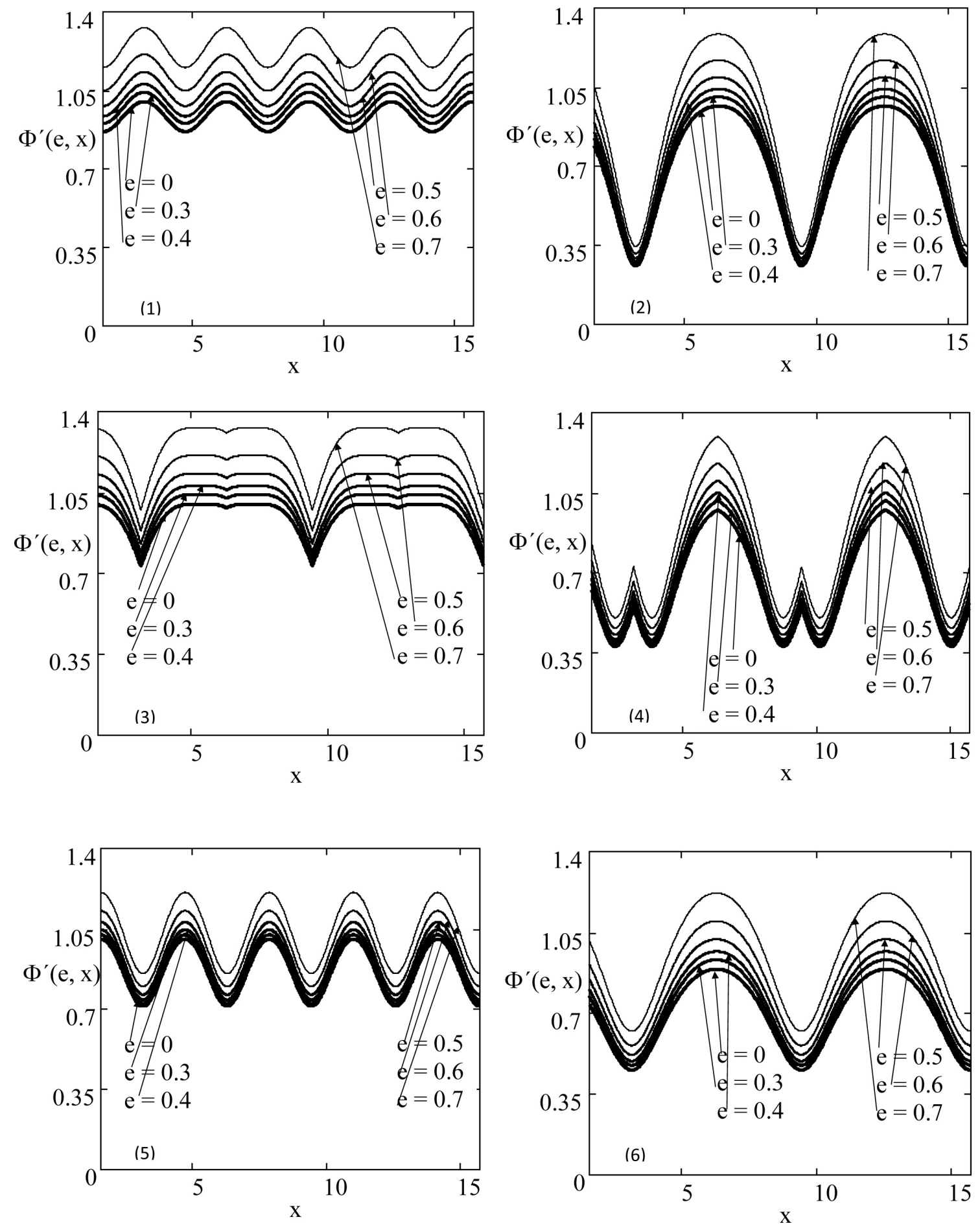

Figure 6. Corrected characteristic phase profiles versus the time $(x \equiv \Omega t)$, viewed at the following position angles $(\theta, \alpha, \phi)$ : (1) $\left(45^{0}, 30^{0}, 0^{0}\right) ;(2)\left(45^{0}, 30^{0}, 90^{0}\right) ;(3)\left(45^{0}, 30^{0}, 135^{0}\right) ;(4)\left(45^{0}, 30^{0}, 60^{0}\right) ;(5)\left(45^{0}, 90^{0}, 90^{0}\right)$; (6) $\left(45^{0}, 18^{0}, 90^{0}\right)$. 

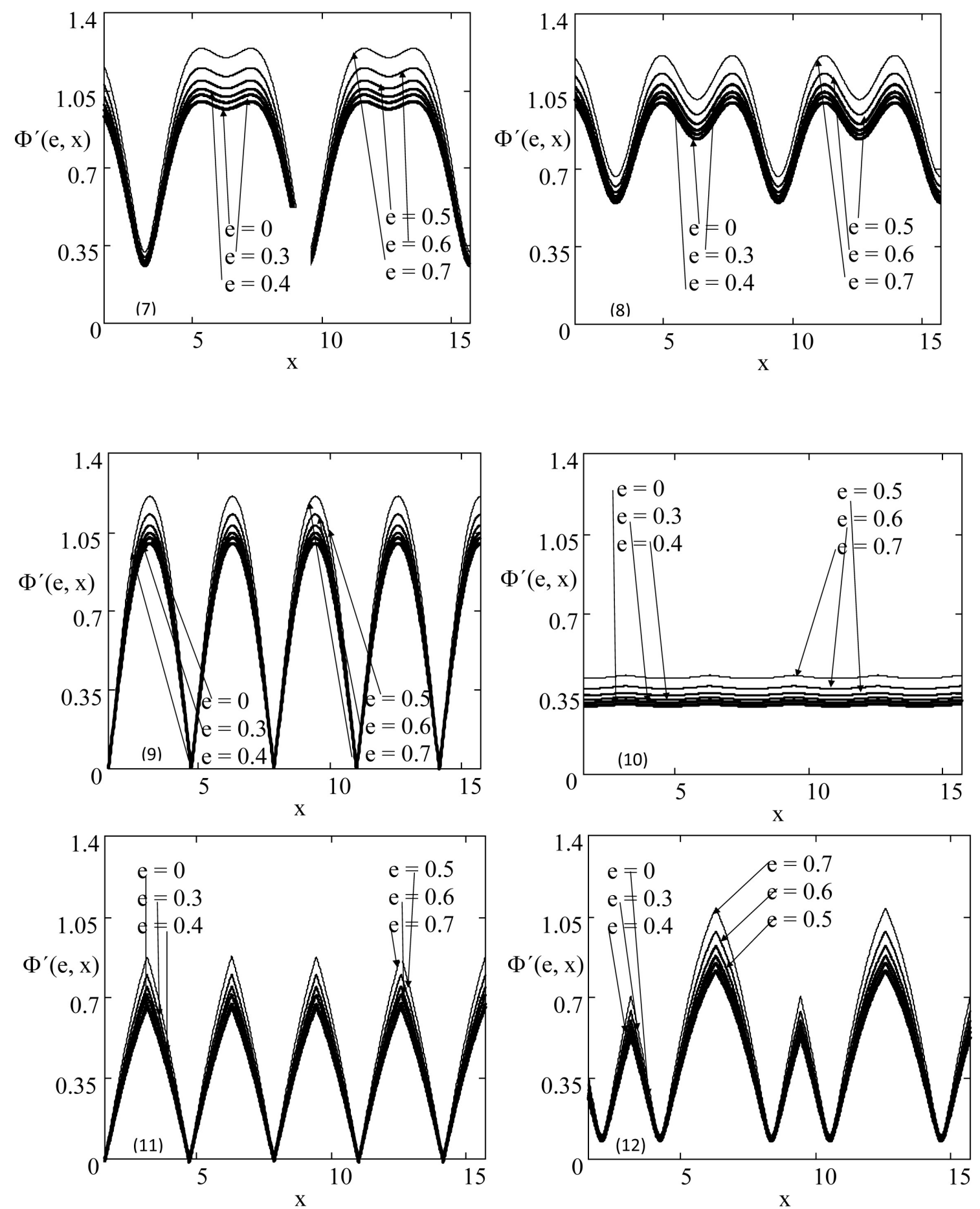

Figure 7. Corrected characteristic phase profiles versus the time $(x \equiv \Omega t)$, viewed at the following position angles $(\theta, \alpha, \phi): \quad(7)\left(45^{0}, 60^{0}, 90^{0}\right)$; (8) $\left(45^{0}, 78.3^{0}, 90^{0}\right)$; (9) $\left(90^{0}, 90^{0}, 180^{0}\right)$; (10) $\left(90^{0}, 0.6^{0}, 72^{0}\right)$; (11) $\left(90^{0}, 30^{0}, 60^{0}\right)$; (12) $\left(72^{0}, 30^{0}, 60^{\circ}\right)$. 

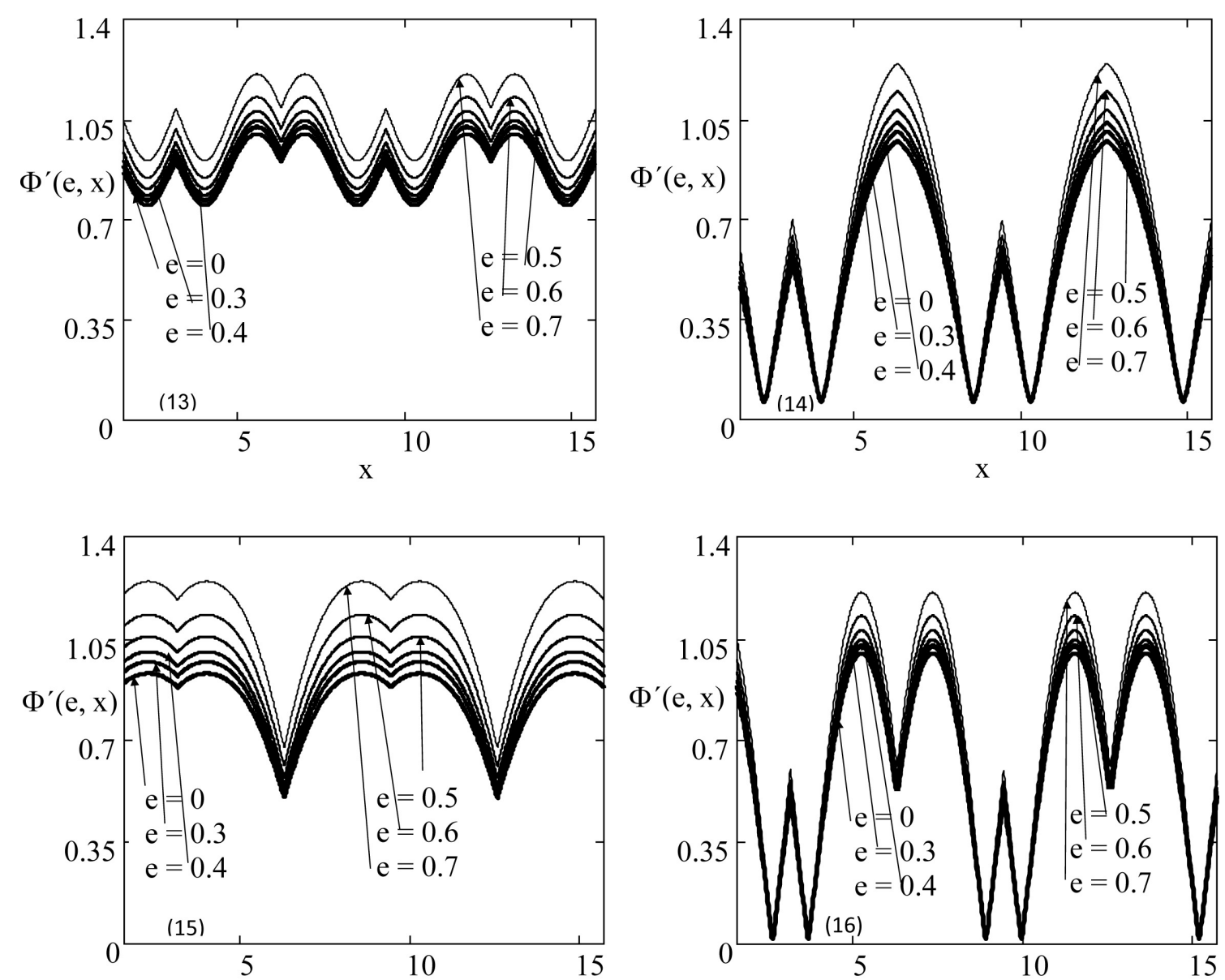

Figure 8. Corrected characteristic phase profiles versus the time $(x \equiv \Omega t)$, viewed at the following position angles $(\theta, \alpha, \phi)$ : (13) $\left(60^{\circ}, 90^{\circ}, 60^{0}\right) ;(14)\left(60^{\circ}, 45^{0}, 60^{\circ}\right) ;(15)\left(60^{0}, 153^{0}, 60^{0}\right) ;(16)\left(1.2^{0}, 90^{0}, 60^{0}\right)$. 


\section{References}

1. G. Ter-Kazarian, "On the Physical Nature of the Source of Ultraluminous X-ray Pulsations", Astrophys. E6 Space Sci., vol. 361, issue 1, pp.20, DOI 10.1007/s10509-015-2604-0, 2016.

2. G. Ter-Kazarian, "Rotating black holes in microscopic theory: the implications for periodic source M82X-2", Advances in Astrophys., Vol.1, issue 3, pp.21, 2016.

3. H. Feng \& R. Soria, "Ultraluminous X-ray sources in the Chandra and XMM-Newton era", New Astron. Rev., vol. 55, Issue 5, pp. 166-183, 2011.

4. J.-F. Liu, J.N. Bregman, Y. Bai, S. Justham \& F. Crowther, "Puzzling accretion onto a black hole in the ultraluminous X-ray source M 101 ULX-1", Nature, vol. 503, Issue 7477, pp. 500-503, 2013.

5. D.R. Pasham, T.E. Strohmayer \& R.F. Mushotzky, "A 400-solar-mass black hole in the galaxy M82", Nature, vol. 513, Issue 7516, pp. 74-76, 2014.

6. J. Poutanen, G. Lipunova, S. Fabrika, A.G. Butkevich, \& P. Abolmasov, Mon. Not. of Roy. Astron. Soc., vol. 377, Issue 3, pp. 1187-1194, 2007.

7. N. McCrady, A.M. Gilbert \& J.R. Graham, "Kinematic Masses of Super-Star Clusters in M82 from HighResolution Near-Infrared Spectroscopy", Astrophys. J., vol. 596, Issue 1, pp. 240-252, 2003.

8. M. Bachetti et al. (24 authors), "An ultraluminous X-ray source powered by an accreting neutron star", Nature, vol. 514, Issue 7521, pp. 202-204, 2014.

9. G. Wiktorowicz, M. Sobolewska, A. Sadowski, K. Belczynski, "Nature of the Extreme Ultraluminous X-Ray Sources", Astrophysical Journal, vol. 810, Issue 1, article id. 20, 8 pp., 2015.

10. A.A. Mushtukov, V.F. Suleimanov, S.S. Tsygankov, J. Poutanen, "On the maximum accretion luminosity of magnetized neutron stars: connecting X-ray pulsars and ultraluminous X-ray sources", Mon. Not. of Roy. Astron. Soc., vol. 454, Issue 3, pp.2539-2548, 2015.

11. D. Simone; P. Rosalba, S. Luigi, "NuSTAR J095551+6940.8: a highly magnetized neutron star with superEddington mass accretion", Mon. Not. of Roy. Astron. Soc., vol. 449, Issue 2, pp.2144-2150, 2015.

12. Y.Y. Pan, L.M. Song, C. M. Zhang, H. Tong, "The magnetic field evolution of ULX NuSTAR J095551+6940.8 in M82âĂ ̌a legacy of accreting magnetar", [astro-ph.HE]/1510.08597], 2016.

13. D. Simone, P. Rosalba; P. Alessandro; B. Enrico; S. Luigi, "The accretion regimes of a highly magnetized NS: the unique case of NuSTAR J095551+6940.8", Mon. Not. of Roy. Astron. Soc., vol. 457, Issue 3, pp.3076-3083, 2016.

14. A. King, J.-P. Lasota, "ULXs: Neutron stars versus black holes", Mon. Not. of Roy. Astron. Soc. Lett., vol. 458, Issue 1, pp.L10-L13, 2016.

15. G. Ter-Kazarian, "Ultra-high energy neutrino fluxes from supermassive AGN black holes", Astrophys. ES Space Sci., vol. 349, pp 919-938, 2014.

16. G. Ter-Kazarian, "Growth of accreting supermassive black hole seeds and neutrino radiation", J. of Astrophysics, vol. 2015, Article ID 205367, p.1, 2015. http://dx.doi.org/10.1155/2015/205367

17. E. Treister, P. Natarajan, D. B. Sanders, C. M. Urry, K. Schawinski, and J. Kartaltepe, "Major Galaxy Mergers and the Growth of Supermassive Black Holes in Quasars", Science, vol. 328, pp. 600-602, 2010.

18. M. Volonteri, G. Lodato, P. Natarajan, "The evolution of massive black hole seeds", Mon. Not. of Roy. Astron. Soc., vol. 383, pp. 1079-1088, 2008.

19. M. Volonteri, P. Natarajan, "Journey to the MBH-s relation: the fate of low-mass black holes in the Universe", Mon. Not. of Roy. Astron. Soc., vol. 400, pp. 1911-1918, 2009.

20. P. Natarajan, "The formation and evolution of massive black hole seeds in the early Universe", in it Fluid Flows to Black Holes: A Tribute to S Chandrasekhar on his Birth Centenary. Edited by Saikia D J et al. Published by World Scientific Publishing Co. Pte. Ltd., ISBN \#9789814374774, pp. 191-206, 2011.

21. E. Treister, \& C.M. Urry, "The Cosmic History of Black Hole Growth from Deep Multiwavelength Surveys", Advances in Astronomy, vol. 2012, id. 516193, 2012.

22. A. J. Barger, L. L. Cowie, R. F. Mushotzky, Y. Yang, W.-H. Wang, A. T. Steffen, and P. Capak, "The Cosmic Evolution of Hard X-Ray-selected Active Galactic Nuclei", Astrophys. J., vol. 129, pp. 578-609, 2005.

23. S. M. Croom, G. T. Richards, T. Shanks, B. J. Boyle, M. A. Strauss, A. D. Myers, R. C. Nichol, K. A. Pimbblet, N. P. Ross, D. P. Schneider, R. G. Sharp, and D. A. Wake, "The 2dF-SDSS LRG and QSO survey: the QSO luminosity function at $0.4<\mathrm{z}<2.6$ ", Mon. Not. of Roy. Astron. Soc., vol. 399, pp. 1755-1772, 2009.

24. W. Krivan, P. Laguna, P. Papadopoulos and N. Andersson, "Dynamics of perturbations of rotating black holes", Phys. Rev. D, Vol. 56, Issue 6, pp.3395-3404, 1997; arXiv:9702048[gr-qc]

25. C.M. Will, Living Rev. Relativity, vol. 17, pp.4, 2014. http://www.livingreviews.org/lrr-2014-4 doi:10.12942/lrr-2014-4; arxiv:1403.7377[gr-qc]

26. R. A. Hulse \& J. H. Taylor, "Discovery of a pulsar in a binary system", Astrophys. J., vol. 195, pp. L51-L53, 1975. 
27. J. H. Taylor \& J. M. Weisberg, Astrophys. J., "A new test of general relativity - Gravitational radiation and the binary pulsar PSR 1913+16", vol. 253, pp. 908-920, 1982.

28. D.Christodoulou \& S.-T. Yau, in Isenberg, J.A., ed., Mathematics and General Relativity, Proc. of the AMSIMS-SIAM Joint Summer Research Conference (1986), vol. 71 of Contemporary Mathematics, 9âĂŞ14, (American Mathematical Society, Providence, U.S.A., 1988.

29. L.B. Szabados, Liv. Rev. Relat., "Quasi-Local Energy-Momentum and Angular Momentum in GR: A Review Article", vol. 7, Issue 1, article id.4, pp.140, 2004.; http://www.livingreviews.org/lrr-2004-4

30. R. Bartnik, in Proc. of the Fifth Marcel Grossmann Meeting on recent developments in theoretical and experimental general relativity, gravitation and relativistic field theories, eds. D.G. Blair and M.J. Buckingham, World Scientific, Singapore; River Edge, U.S.A., pp.399, 1989.

31. R. Bartnik, "New definition of quasilocal mass", Phys. Rev. Lett., vol. 62, Issue 20, pp.2346-2348, 1989.

32. S.W. Hawking, J. Math. Phys., "Gravitational Radiation in an Expanding Universe", vol. 9, p.598-604, 1968.

33. R. Geroch, "Energy Extraction", Ann. N.Y. Acad. Sci., Vol. 224, pp.108, 1973.

34. R. Penrose, "Quasi-Local Mass and Angular Momentum in General Relativity", Proc. R. Soc. London, Ser. $A$, vol. 381, Issue 1780 , pp. 53-63, 1982.

35. R. Penrose \& W. Rindler, Spinors and space-time, vol. 2: Spinor and twistor methods in space-time geometry, Cambridge Monographs on Mathematical Physics, Cambridge University Press, Cambridge, U.K.; New York, U.S.A., 1986.

36. A.J. Dougan \& L.J. Mason, "Quasilocal mass constructions with positive energy", vol. 67, Issue 16, pp.21192122,1991

37. J.D. Brown \& J.M. York, "Microcanonical functional integral for the gravitational field", Phys. Rev. D, vol. 47, Issue 4, pp.1420-1431, 1993.; arXiv:9209012[gr-qc]

38. S.W. Hawking, "Wormholes in spacetime", Phys. Rev. D, vol. 37, Issue 4,pp.904-910, 1988.

39. S. Coleman, "Why there is nothing rather than something: A theory of the cosmological constant", vol. 310, Issue 3, p. 643-668, 1988.

40. T. Dray \& G. âĂŹt Hooft, "The gravitational shock wave of a massless particle", Nucl. Phys. B, vol. 253, pp. 173-188, 1985.

41. T. Dray and G. âẮ́t Hooft, "The effect of spherical shells of matter on the Schwarzschild black hole", Commun. Math. Phys., vol. 99, Issue 4, pp.613-625, 1985.

42. T.W. Baumgarte, S.L. Shapiro, "General Relativistic Magnetohydrodynamics for the Numerical Construction of Dynamical Spacetimes", Astrophys. J., Volume 585, Issue 2, pp. 921-929, 2003.

43. S.W.Hawking \& G.F.R. Ellis, The large Scale Structure of Space-time, Cambrige University press, Cambrige, England, 1973.

44. B. Carter, in An Einstein Centenary Survay, ed. S.W. Hawking, W.Israel, p. 294, Cambridge Univ. press, Cambridge, 1979.

45. J.M. Bardeen, B.Carter, S.W.Hawking, "The four laws of black hole mechanics", vol. 31, Issue 2, pp.161-170, 1973.

46. J.D.Bekenstein, "Black Holes and Entropy", Phys. Rev. D, vol. 7, Issue 8, pp. 2333-2346, 1973.

47. J.D.Bekenstein, "Generalized second law of thermodynamics in black-hole physics", Phys. Rev. D, vol. 9, Issue 12, pp. 3292-3300, 1974.

48. J.D.Bekenstein, "Universal upper bound on the entropy-to-energy ratio for bounded systems", Phys. Rev. D, Volume 23, Issue 2, pp.287-298, 1981.

49. J.D.Bekenstein, "Black holes and everyday physics", Gen. Relativ. Gravit., vol. 14, pp. 355-359, 1982.

50. N. D. Birrell \& P. C. W. Davies, Quantum Fields in Curved Space, Cambridge Iniv. Press, 1982.

51. R. Kerner \& R. B. Mann, "Fermions tunnelling from black holes", Class. Quant. Grav., vol. 25, Issue 9, id. 095014, 2008.

52. P.K. Townsend, "Black holes", Lecture notes, 1997; arxiv:9707012[gr-qc].

53. K. Fredenhagen, "Gravity Induced Noncommutative Spacetime", Rev. in Math. Phys., vol. 7, Issue 04, pp. 559-565, 1995.

54. S. Carlip, "Near-Horizon Conformal Symmetry and Black Hole Entropy", Phys. Rev. Lett., vol. 88, Issue 24, id. 241301, 2002.

55. M.I. Park, "The Hamiltonian Dynamics of Bounded Spacetime and Black Hole Entropy: The Canonical Method", Nucl. Phys. B, vol.634, pp.:339-369, 2002.

56. J.D. Brown, J.D.E. Creighton, R. Mann, "Temperature, energy, and heat capacity of asymptotically anti-de Sitter black holes", Phys. Rev. D, vol. 50, Issue 10, pp.6394-6403, 1994.

57. S.A. Hayward, "Unified first law of black-hole dynamics and relativistic thermodynamics", Class. Quantum Grav., vol. 15, Issue 10, pp. 3147-3162, 1998.

58. G. 't Hooft, arxiv:9310026[gr-qc] 
59. L. Susskind, "The world as a hologram", J. Math. Phys., vol. 36, Issue 11, pp.6377-6396, 1995; [hepth/9409089].

60. R.M. Wald, Living Rev. Relativity, vol. 4, lrr-2001-6, 2001. URL (cited on 29 January 2004): http://www.livingreviews.org/lrr-2001-6.

61. R. Bousso, "The holographic principle", Rev. Mod. Phys., vol. 74, Issue 3, pp. 825-874, 2002; arxiv:0203101[hepth].

62. R. Bousso, "Holography in general space-times", J. High Energy Phys., Issue 06, id. 028, 1999; arXiv:9906022[hep-th].

63. É.É.Flanagan, D. Marolf \& R.M. Wald, "Proof of classical versions of the Bousso entropy bound and of the generalized second law", Phys. Rev. D, vol. 62, Issue 8, id.084035, 2000; arXiv:9908070[hep-th]

64. L.B. Szabados, "Quasi-local holography and quasi-local mass of classical fields in Minkowski spacetime", Class. Quantum Grav., vol. 22, Issue 5, pp. 855-878 (2005); arXiv:0411148[gr-qc]

65. J. M. Maldacena, "The Large N Limit of Superconformal Field Theories and Supergravity", Adv. Theor. Math. Phys., vol. 2, p. 231, 1998; arxiv:9711200[hep-th].

66. O. Aharony, S. S. Gubser, J. M. Maldacena, H. Ooguri and Y. Oz, "Large N field theories, string theory and gravity", Phys. Rept., vol. 323, Issue 3, pp. 183-386, 2000; [hep-th/9905111].

67. S. S. Gubser, I. R. Klebanov and A. W. Peet, "Entropy and temperature of black 3-branes", Phys. Rev. D, vol. 54, Issue 6, pp.3915-3919, 1996; arxiv9602135[hep-th]

68. W. Israel, "Thermo-field dynamics of black holes", Phys. Lett. A, vol. 57, Issue 2, pp. 107-110, 1976.

69. G. 't Hooft, "On the quantum structure of a black hole", Nucl. Phys. B, vol. 256, pp. 727-745 1985.

70. R. Brustein, M.B.Einhorn, A. Yarom, "Entanglement interpretation of black hole entropy in string theory", J. of High Energy Phys., Issue 01, id. 098, 2006. (2006)

71. T. Nishioka, Sh.Ryu, T. Takayanagi, "Holographic entanglement entropy: an overview", J. of Phys. A: Math. and Theor., vol. 42, Issue 50, article id. 504008, 35 pp., 2009. arxiv:0905.0932[hep-th].

72. G. Ter-Kazarian, S. Shidhani \& L. Sargsyan, "Neutrino Radiation of The AGN Black Holes", Astrophys. \&6 Space Sci., Vol.310, pp. 93-110, 2007.

73. A. Komar, "Covariant Conservation Laws in General Relativity", Phys.rev., vol. 113, p.934, 1959.

74. D.C. Robinson, "Uniqueness of the Kerr black hole", Phys. Rev. Lett., vol. 34, pp. 905, 906, 1975. 